\title{
DIFERENÇAS FLORÍSTICAS E ESTRUTURAIS ENTRE FITOFISIONOMIAS DO CERRADO EM ASSIS, SP, BRASIL ${ }^{1}$
}

\author{
Eduardo da Silva Pinheiro² e Giselda Durigan ${ }^{3}$
}

\begin{abstract}
RESUMO - A classificação das fitofisionomias tem-se constituído em desafio entre os pesquisadores da vegetação de cerrado, seja por não haver critérios florísticos ou quantitativos claros para sua separação, seja pelas alterações que sofrem ao longo do tempo. O objetivo deste estudo foi caracterizar três tipos fitofisionômicos de cerrado na Estação Ecológica de Assis, bem como verificar se são florística e, ou, estruturalmente distintos, buscando-se as melhores variáveis para caracterizá-los. A área amostral compreendeu 30 parcelas permanentes de 20 x $50 \mathrm{~m}$, sendo 10 parcelas para cada um dos tipos fisionômicos: cerrado típico, cerrado denso e cerradão, em que foram identificadas e medidas as árvores com diâmetro à altura do peito $\geq 5 \mathrm{~cm}$. As três fitofisionomias de cerradoestudadas mostraram-se estruturalmente distintas em classes de área basal, cobertura de copas e altura das maiores árvores. O melhor descritor para classificar as fitofisionomias, por ser facilmente mensurável e pouco variável com o critério de inclusão, é a área basal $\left(\mathrm{m}^{2} \mathrm{ha}^{-1}\right)$. Floristicamente, as fitofisionomias savânicas (cerradotípico e cerrado denso) não se diferenciam, quer seja analisando apenas a presença e ausência das espécies, quer seja analisando a importância relativa das espécies na comunidade (fitossociologia). Em síntese, há três fitofisionomias distintas, mas a flora se diferencia apenas entre o cerradão e as fitofisionomias savânicas. A análise das espécies exclusivas de cada fitofisionomia quanto à tolerância à sombra, com base na literatura, indicou que a baixa disponibilidade de luz sob as copas no cerradão pode ter sido o fator condicionante da diferenciação entre esta e as demais fitofisionomias do cerrado lato sensu.
\end{abstract}

Palavras-chave: Classificação fisionômica, Descritores estruturais, Savana, tipos fisionômicos e similaridade florística.

\section{FLORISTIC AND STRUCTURAL DIFFERENCES AMONG CERRADO PHYTOPHYSIOGNOMIES IN ASSIS, SP, BRAZIL}

\begin{abstract}
Classification of physiognomies is a challenge for many cerrado vegetation researches either because there are no clear floristic and quantitative criteria for its classification and because the changes it had over come over the years. The objective of this study was to charactherize three phytophysiognomies types in the cerradovegetation (Brazilian savannah) in the Estação Ecológica de Assis as well as to verify if they are floristically or structurally disticnts searching for better variables to classify them. The sample consisted of 30 permanent plots with $20 \times 50 \mathrm{~m}$, being 10 plots for each one of the physiognomy types: typical cerrado, dense cerrado and cerradão, where trees with diameter at breast height $\geq 5 \mathrm{~cm}$. The three studied phytophysiognomies were structuraly distintics in terms of basal area, canopy cover, and average height and diameter of the tallest trees. Basal area $\left(\mathrm{m}^{2} \mathrm{ha}^{-1}\right)$ was considered the best descriptor to classify the physiognomies of the cerrado vegetation because it is to easy measure and less variable with the inclusion criteria. In terms of floristic composition, savannah phytophysiognomies (typical cerrado and woodland cerrado) are not different from each other either by analyzing only the presence and absence of species or by analyzing the relative importance of the species in the community. Briefily, there are three distinct phytophysiognomies but the flora is different only between woodland cerrado and savannah phytophysiognomies. By analyzing the exclusive species of each physiognomy in relation to tolerance shade tolerance, it was suggested based in the literature that the low light availability under the canopies in the woodland cerrado might have been the conditioning factor of the differentiation between it and the other cerrado physiognomies.
\end{abstract}

Keywords: Physiognomyc classification, Savannah structural descriptors, Vegetation types and floristic similarity

\footnotetext{
${ }^{1}$ Recebido em 20.08.2009 e aceito para publicação em 15.11.2011.

${ }^{2}$ Universidade Federal do Amazonas, Manaus - AM, Brasil. E-mail: <pinheiro@ufam.edu.br>.

${ }^{3}$ Floresta Estadual de Assis, Instituto Florestal, Assis, SP, Brasil. E-mail: <giselda@femanet.com.br>.
} 


\section{INTRODUÇÃO}

O cerrado está entre as savanas mais ricas do planeta (RATTER et al., 2006) devido à sua riqueza biológica, entretanto vem sendo submetido a forte pressão antrópica (AGUIAR et al., 2004). A combinação de alta riqueza e grau de ameaça levou à sua inclusão entre as 25 áreas consideradas críticas para a conservação da biodiversidade no mundo (MYERS et al., 2000). Segundo Machado et al. (2004), apenas 20\% da cobertura original do cerrado permanece intacta e somente $2,2 \%$ de sua área original de ocorrência está inserida em áreas protegidas.

Compreendendo ampla variação de fitofisionomias da vegetação, desde campos com arbustos esparsos e árvores pequenas até uma formação florestal fechada, com altura do dossel de 8 a 13 m (RATTER et al., 2006), a difícil classificação das fitofisionomias do Cerrado tem sido objeto de estudo de diferentes autores (EITEN, 1972, 1979; COUTINHO, 1978; RIBEIRO et al., 1983, 1985; RIBEIRO;WALTER, 1998, 2008; OLIVEIRA-FILHO; RATTER, 2002; WALTER, 2006). Ainda que a classificação tenha sido objeto de várias pesquisas, raramente são utilizados métodos de classificação baseados em descritores quantitativos, podendo ser citado o estudo pioneiro de Ribeiro et al. (1985) em áreas de cerrado no Distrito Federal.

Ribeiro e Walter (1998, 2008) propuseram uma classificação para diferenciar os principais tipos fisionômicos do cerrado, de acordo com a estrutura, formas de crescimento dominantes, possíveis mudanças estacionais, fatores edáficos e composição florística. Ainda que essa classificação não se baseie em dados quantitativos, tem sido utilizada por diversos autores em decorrência de seu pragmatismo (DURIGAN et al., 2004a; RATTER et al., 2006). No total, são descritos 11 tipos fisionômicos gerais, compostos por formações florestais (em que as árvores formam estrato contínuo), savânicas (árvores esparsas sobre estrato graminoso) e campestres (árvores ausentes). Subdivididos pelos autores, esses tipos gerais resultaram em 23 tipos vegetacionais distintos, sendo o cerrado sentido restrito, por exemplo, subdividido em cerrado típico, cerrado denso, cerrado ralo e cerrado rupestre.

Ribeiro e Walter (1998) destacaram as dificuldades para uma classificação da vegetação do cerrado, uma vez que tais tentativas tendem a adotar critérios e escalas diferentes, baseadas em princípios ou origens distintas.
Para Oliveira-Filho e Ratter (2002), a principal complicação para essa classificação se deve ao fato de que qualquer categoria dentro de uma classificação fisionômica da vegetação do cerrado é, na verdade, um segmento de uma série vegetacional contínua. A classificação das fitofisionomias tem-se constituído em desafio entre os pesquisadores da vegetação do cerrado, seja por não haver nitidez em sua separação, seja pelas alterações que a vegetação sofre ao longo do tempo em um mesmo local (alterações sucessionais), devido à frequência e intensidade de impactos como o fogo e o pastoreio (DURIGAN et al., 2002). Essas transformações têm sido documentadas na região deste estudo (DURIGAN; RATTER, 2006; DURIGAN, 2006; PINHEIRO; DURIGAN, 2009), mostrando tendência ao desaparecimento das fitofisionomias savânicas e campestres, substituídas pelo cerradão.

Desenvolver critérios claros para a classificação das fitofisionomias é necessário para dar suporte tanto ao mapeamento de tipos de vegetação, em escala detalhada, quanto para estudos ecológicos que, muitas vezes, dependem da exata descrição da estrutura da comunidade vegetal para que sejam compreendidos. Ainda que os tipos fisionômicos façam parte de um continuum, o que se propôs neste estudo foi identificar as classes com base em variáveis mensuráveis, de modo que possam ser mais facilmente reconhecidas.

O objetivo deste estudo foi comparar, florística e estruturalmente, parte das fitofisionomias de cerrado lato sensu com base naquelas que ocorrem na Estação Ecológica de Assis, SP, partindo-se dos tipos mencionados na classificação detalhada proposta por Ribeiro e Walter (1998), na busca de explicar as diferenças eventualmente existentes entre elas.

\section{MATERIAL E MÉTODOS}

A Estação Ecológica de Assis (EEcA), que contém um dos últimos remanescentes de cerrado do Estado de São Paulo, tem sido objeto de estudos florísticos e ecológicos recentes (DURIGAN et al., 1999; BRANDO; DURIGAN, 2004; ROSSATTO et al., 2008), nos quais é mencionada a existência de fitofisionomias distintas no interior da área protegida. Essa unidade ocupa uma área de 1.760,64 ha, no Município de Assis, Estado de São Paulo, entre as coordenadas 22 $33^{\circ} 20^{\prime \prime}$ e $22^{\circ} 37^{\prime} 41^{\prime \prime}$ de latitude Sul e 50²4’4,8" e 50²1’27" de longitude Oeste de Greenwich. 
As diferentes fitofisionomias da unidade de conservação, incluindo tipos vegetacionais ripários, foram mapeadas por Pinheiro e Durigan (2009), por meio de imagens do satélite QuickBird, de 04/07/2006, tendo sido classificadas, em campo, segundo os tipos propostos por Ribeiro e Walter (1998). Entre essas fitofisionomias, que incluem tipos vegetacionais ripários, foram objeto deste estudo as três que ocupavam as áreas de interflúvio, quais sejam: o cerradão (fitofisionomia florestal), correspondente a 91,43\% da área protegida; as duas fitofisionomias savânicas - o cerrado denso, correspondente a $2,56 \%$; e o cerrado típico, que cobre atualmente $0,84 \%$ da unidade de conservação. O cerrado típico e o cerrado denso, segundo a classificação de Ribeiro e Walter (1998), são subtipos do cerrado sentido restrito. As fitofisionomias estudadas foram reconhecidas pelos seguintes atributos: (a) cerrado típico: estrato arbóreo descontínuo, piso parcialmente revestido por estrato graminoso; (b) cerrado denso: estrato arbóreo descontínuo e ausência de gramíneas, sendo o estrato inferior formado por arbustos e subarbustos em alta densidade; e (c) cerradão: estrato arbóreo contínuo.

Para amostragem, visando à caracterização florística e estrutural da comunidade arbórea, foi utilizado o método deparcelas (MÜELLER-DOMBOIS; ELLENBERG 1974), com 10 parcelas representando cada fitofisionomia (30 parcelas no total). As parcelas foram distribuídas de modo a representar toda a área ocupada por cada uma das três fitofisionomias mapeadas. A área de cada parcela foi de $1.000 \mathrm{~m}^{2}$ (20 x $50 \mathrm{~m}$ ), conforme proposto por Felfili et al. (1994), para facilidade operacional da amostragem de vegetação do cerrado. As coordenadas dos vértices de cada parcela foram obtidas por meio de um aparelho GPS Trimble Pro- $X R$, utilizando-se o método DGPS com coordenadas pós-processadas, tendo sido o erro médio de posicionamento inferior a $0,5 \mathrm{~m}$. As parcelas foram sobrepostas na imagem QuickBird (resolução espacial de 0,6 m), de modo a possibilitar a determinação da cobertura de copas, pelo método de linhas adaptado a partir de Canfield (1941) e Melo et al. (2007).

Foram amostrados todos os indivíduos vivos de porte arbóreo, adotando-se como critério de inclusão o Diâmetro do Tronco à Altura do Peito (DAP) igual ou superior a $5 \mathrm{~cm}$. Todas as árvores amostradas foram numeradas com etiqueta metálica. Em árvores com troncos múltiplos, cada um dos troncos foi medido separadamente, desde que pelo menos um tivesse o DAP $\geq 5 \mathrm{~cm}$. Para cálculo da área da secção do tronco do indivíduo, foram somadas as áreas seccionais de todos os troncos medidos. Os dados adquiridos em campo foram: DAP, medido com fita diamétrica; altura total da árvore, medida com o auxílio de régua dendrométrica telescópica, desde a base até o ramo mais alto; e identificação botânica.

As espécies amostradas foram classificadas conforme proposto pelo Angiosperm Phylogeny Group (APG II) (APG 2003). No caso de algumas espécies que não puderam ser identificadas em campo, foi coletado material botânico para comparação com exsicatas depositadas na Coleção Botânica da Floresta Estadual de Assis, no Herbário D. Bento Pickel, do Instituto Florestal do Estado de São Paulo, ou por consulta a especialistas. As espécies foram classificadas quanto à tolerância à sombra, com base em Durigan et al. (2004b).

Em cada fitofisionomia foram calculados os parâmetros fitossociológicos usuais (densidade, frequência e dominância das espécies), de acordo com os métodos compilados por Durigan (2003). Especialmente para caracterizar cada fitofisionomia pelas suas espécies mais representativas, foi utilizado o índice de valor de importância (IVI), o qual inclui nos cálculos valores relativos de dominância, densidade e frequência das espécies, a saber:

$$
\mathrm{IVI}_{\mathrm{i}}=\mathrm{DR}_{\mathrm{i}}+\mathrm{DoR}_{\mathrm{i}}+\mathrm{FR}_{\mathrm{i}}
$$

em que $\mathrm{IVI}_{\mathrm{i}}=$ índice de valor de importância da espécie $\mathrm{i}, \mathrm{DR}_{\mathrm{i}}=$ densidade relativa da espécie i (\%), $\mathrm{DoR}_{\mathrm{i}}=$ dominância relativa da espécie i e $\mathrm{FR}_{\mathrm{i}}=$ frequência relativa da espécie i (\%).

Foram consideradas como mais representativas, para esta análise, as 10 espécies com maior IVI, com base no fato de que estas correspondem a pelo menos a metade do somatório de IVI de todas as espécies em cada fitofisionomia e, portanto, dominam as comunidades.

Em cada fitofisionomia foram calculados o índice de diversidade de Shannon (H') com base neperiana (PIELOU, 1975) e o índice de equabilidade de Pielou (e) (PIELOU, 1966).

$$
\mathrm{H}^{\prime}=-\sum_{\mathrm{i}=1}^{\mathrm{n}} \mathrm{P}_{\mathrm{i}} \ln \mathrm{P}_{\mathrm{i}}
$$

em que H' = índice de diversidade de Shannon, $\mathrm{P}_{\mathrm{i}}=\mathrm{n}_{\mathrm{i}} / \mathrm{N}, \mathrm{n}_{\mathrm{i}}=$ número de árvores da espécie i e $\mathrm{N}=$ número total de árvores amostradas.

$$
e=\mathrm{H}^{\prime} / \mathrm{H}^{\prime}{ }_{\text {máximo }}
$$

Revista Árvore, Viçosa-MG, v.36, n.1, p.181-193, 2012 
em que $e$ = índice de equabilidade de Pielou (e), H' = índice de diversidade de Shannon e s = número total de espécies amostradas.

As espécies exclusivas e comuns às fitofisionomias de cerrado estudadas foram agrupadas em um diagrama de Venn (VENN, 1880), apresentado por Zar (1999) entre as ferramentas para estudos de ecologia de comunidades, ilustrando as relações entre os conjuntos de espécies amostradas nas diferentes fisionomias. Foi analisada a similaridade florística entre as fitofisionomias, por meio de classificação aglomerativa das parcelas pelo método de médias aritméticas (Unweighted Pair Groups Method using Arithmetic Averages - UPGMA), com o uso do coeficiente de similaridade de Jaccard (JAMES; MCCULLOCH, 1990; BELBIN; MCDONALD, 1993; BALDUINO et al., 2005), pela fórmula a seguir.

$$
\mathrm{Cj}=\mathrm{c} /(\mathrm{a}+\mathrm{b}-\mathrm{c}),
$$

em que $\mathrm{Cj}$ = coeficiente de similaridade de Jaccard, $\mathrm{a}=$ número total de espécies presentes em uma das amostras, b = número total de espécies presentes na outra amostra e c = número de espécies comuns a ambas as amostras.

Com base em Müeller-Dombois e Ellenberg (1974), foram consideradas floristicamente distintas as fitofisionomias quando o valor do índice não ultrapassava 0,25 , ou seja, quando menos de $25 \%$ das espécies amostradas eram comuns às duas fisionomias em comparação.

Para as comparações de estrutura, foram calculadas a área basal, a densidade e a altura média de todas as árvores dentro de cada parcela. Considerando-se que a altura e o diâmetro são variáveis que não seguem distribuição normal dentro das parcelas ou fisionomias e, portanto, não são bem representadas pelos valores médios, foi calculada a média dos cinco maiores valores de altura e diâmetro equivalente $(d g)$ em cada parcela. O diâmetro equivalente $(d g)$ é um valor único de DAP obtido a partir da área basal de árvores com troncos múltiplos, que melhor representa o porte das árvores, ou seja:

$$
\operatorname{dg}=\sqrt{\frac{4}{\pi}} g
$$

em que dg = diâmetro equivalente $(\mathrm{cm})$ e g = área basal do indivíduo $\left(\mathrm{cm}^{2}\right)$.
Testes estatísticos foram aplicados para comparação dos valores médios das variáveis estruturais das 10 parcelas de cada fitofisionomia. Para verificar a normalidade e a homocedasticidade dos dados, foram aplicados os testes de Shapiro-Wilk e de Barlett. Posteriormente, os dados foram submetidos à análise de variância (ANOVA), adotando-se o nível de significância de $5 \%$. As médias foram comparadas pelo teste de Tukey.

\section{RESULTADOS}

No levantamento florístico de todas as fitofisionomias, somando $30.000 \mathrm{~m}^{2}$ de área amostrada, foram registradas 102 espécies, pertencentes a 69 gêneros e 43 famílias (Tabela 1 ). No cerrado típico foram amostradas 50 espécies, 54 no cerrado denso (incluindo uma espécie exótica invasora - Pinus elliotii) e 71 no Cerradão.

A diversidade florística obtida pelo índice de Shannon ( $\mathrm{H}^{\prime}$ ) foi de 2,82 no cerrado típico (equabilidade de 72\%), 2,72 no cerrado denso (equabilidade de 67\%) e 3,19 no cerradão (equabilidade de $75 \%$ ). O diagrama de Venn (Figura 1) mostra que 23 espécies foram comuns às três fitofisionomias de cerrado na área de estudo. Quanto às espécies exclusivas, foram identificadas 13 no cerrado típico, 6 no cerrado denso e 32 no cerradão (Tabela 1 e Figura 1). Doze espécies foram comuns ao cerrado típico e ao cerrado denso, duas espécies comuns ao cerrado típico e ao cerradão e 14 espécies comuns ao cerrado denso e ao cerradão.

A análise fitossociológica resulta em ordenação distinta das espécies nas diferentes fitofisionomias, com base em sua importância relativa na comunidade. Analisando apenas as 10 espécies de maior valor de importância (Figura 2), verificou-se que há apenas uma troca de posições de algumas espécies entre o cerrado típico e o cerrado denso, sendo que oito espécies foram as mesmas entre as 10 mais importantes de ambos (Copaifera langsdorffii, Dimorphandra mollis, Eriotheca gracilipes, Gochnatia polymorpha, Machaerium acutifolium, Ocotea corymbosa, Stryphonodendron obovatum e Vochysia tucanorum). Por outro lado, no cerradão surgem novas espécies entre as mais importantes, com apenas três ( $C$. langsdorffii, O. corymbosa e V. tucanorum) figurando entre as mais importantes também nas fitofisionomias savânicas, de modo que a distinção dessa fitofisionomia, apontada pela análise florística, se confirma na análise 
Tabela 1 - Famílias e espécies amostradas no cerrado típico, cerrado denso e cerradão, na Estação Ecológica de Assis, SP. *Espécies tolerantes à sombra.

Table 1 - Plant species recorded in the typical cerrado, dense cerrado and woodland cerrado at Assis Ecological Station, state of São Paulo, Brazil. * Shade tolerant species.

\begin{tabular}{|c|c|c|c|c|c|c|}
\hline \multirow[b]{2}{*}{ Família / Espécie } & \multicolumn{4}{|c|}{$\mathrm{N}^{\circ}$ de árvores amostrados } & \multirow[b]{2}{*}{ Cerradão } & \multirow[b]{2}{*}{ Total } \\
\hline & Abreviatura. & Nome popular $\quad C$ & $\begin{array}{l}\text { Cerrado } \\
\text { típico }\end{array}$ & $\begin{array}{c}\text { Cerrado } \\
\text { denso }\end{array}$ & & \\
\hline \multicolumn{7}{|l|}{ Anacardiaceae } \\
\hline Tapirira guianensis Aubl.* & T.guia & peito-de-pomba & 3 & 12 & 51 & 66 \\
\hline \multicolumn{7}{|l|}{ Annonaceae } \\
\hline Annona coriacea Mart. & A.cori & araticum-do-campo & 1 & & & 1 \\
\hline Annona crassiflora Mart. & A.cras & marolo-verdadeiro & 10 & 18 & & 28 \\
\hline Xylopia aromatica (Lam.) Mart. & X.arom & pimenta-de-macaco & 5 & 33 & 132 & 170 \\
\hline Xylopia brasiliensis Spreng.* & X.brasi & pindaíba & & & 1 & 1 \\
\hline \multicolumn{7}{|l|}{ Apocynaceae } \\
\hline Aspidosperma tomentosum Mart. & A.tome & peroba-do-campo & 2 & 1 & & 3 \\
\hline Aquifoliaceae & & & & & 1 & 1 \\
\hline Ilex paraguariensis A. St.-Hil.* & I.para & erva-mate & & & 1 & 1 \\
\hline \multicolumn{7}{|l|}{ Araliaceae } \\
\hline $\begin{array}{l}\text { Scheffera vinosa (Cham. \& Schltdl.) Frodin \& } \\
\text { Fiaschi }\end{array}$ & S.vino & $\begin{array}{l}\text { mandioquinha-do- } \\
\text { cerrado }\end{array}$ & 3 & & & 3 \\
\hline Arecaceae & & & & 2 & 10 & 12 \\
\hline Syagrus romanzoffiana (Cham.) Glassman* & S.roma & jerivá & & 2 & 10 & 12 \\
\hline \multicolumn{7}{|l|}{ Asteraceae } \\
\hline Gochnatia barrosii Cabrera & G.barr & & & 1 & & 1 \\
\hline Gochnatia polymorpha (Less.) Cabrera & G.poly & candeia & 49 & 24 & 5 & 78 \\
\hline $\begin{array}{l}\text { Piptocarpha axillaris (Less.) Baker } \\
\text { miúda }\end{array}$ & P.axil & cambará de folha & & & 1 & 1 \\
\hline Piptocarpha rotundifolia (Less.) Baker & P.rotu & cambará & 6 & 4 & & 10 \\
\hline \multicolumn{7}{|l|}{ Bignoniaceae } \\
\hline $\begin{array}{l}\text { Tabebuia aurea (Silva Manso) Benth \& Hook.f. } \\
\text { ex S.Moore }\end{array}$ & T.aure & $\begin{array}{l}\text { ipê-amarelo-do- } \\
\text { cerrado }\end{array}$ & 1 & & & 1 \\
\hline Tabebuia ochracea (Cham.) Standl & T.ochr & ipê-amarelo-do-campo & 14 & 18 & 2 & 34 \\
\hline \multicolumn{7}{|l|}{ Bombacaceae } \\
\hline Eriotheca gracilipes (K.Schum.) A.Robyns & E.graci & paineira-do-campo & 60 & 28 & 1 & 89 \\
\hline \multicolumn{7}{|l|}{ Burseraceae } \\
\hline Protium heptaphyllum (Aubl.) Marchand* & P.hept & amescla & & & 184 & 184 \\
\hline \multicolumn{7}{|l|}{ Caryocaraceae } \\
\hline Caryocar brasiliense Cambess. & C.brasi & pequi & 2 & 2 & & 4 \\
\hline \multicolumn{7}{|l|}{ Celastraceae } \\
\hline Maytenus robusta Reissek* & M.robu & cafezinho & & & 3 & 3 \\
\hline Plenckia populnea Reissek & P.popu & marmeleiro-do-campo & 3 & & & 3 \\
\hline \multicolumn{7}{|l|}{ Chrysobalanaceae } \\
\hline $\begin{array}{l}\text { Couepia grandiflora (Mart. \& Zucc.) Benth. } \\
\text { ex Hook. f. }\end{array}$ & C.gran & fruta-de-ema & & & 1 & 1 \\
\hline Licania humilis Cham. \& Schltdl & L.hum & & & 1 & & 1 \\
\hline \multicolumn{7}{|l|}{ Clethraceae } \\
\hline Clethra scabra Pers.* & C.scab & guaperê & & & 1 & 1 \\
\hline Clusiaceae & & & 1 & & & 1 \\
\hline Kielmeyera coriacea Mart. \& Zucc. & K.cori & pau-santo & 1 & & & 1 \\
\hline \multicolumn{7}{|l|}{ Combretaceae } \\
\hline Terminalia glabrescens Mart. & T.glab & capitão & & 2 & 28 & 30 \\
\hline \multicolumn{7}{|l|}{ Connaraceae } \\
\hline Connarus suberosus Planch var. suberosus & C.sube & para-tudo & 1 & & & 1 \\
\hline
\end{tabular}


Tabela 1 - Cont.

Table 1 - Cont.

\begin{tabular}{|c|c|c|c|c|c|c|}
\hline \multicolumn{7}{|l|}{ Ebenaceae } \\
\hline \multicolumn{7}{|l|}{ Erythroxylaceae } \\
\hline Erythroxylum suberosum A.St.-Hil. & E.sube & mercúrio-do-campo & 4 & & & 4 \\
\hline Erythroxylum tortuosum Mart. & E.tort & mercúrio-do-campo & & 2 & & 2 \\
\hline \multicolumn{7}{|l|}{ Euphorbiaceae } \\
\hline Alchornea triplinervia (Spreng.) Müll. Arg.* & A.trip & tapiá & & & 2 & 2 \\
\hline Croton floribundus Spreng. & C.flor & capixingui & & & 2 & 2 \\
\hline Mabea fistulifera Mart.* & M.fist & canudo-de-pito & & & 62 & 62 \\
\hline Maprounea guianensis Aubl.* & M.guia & bonifácio & & 1 & 18 & 19 \\
\hline Pera obovata (Klotzsch) Baill.* & P.obov & tabocuva & 1 & 7 & 71 & 79 \\
\hline \multicolumn{7}{|l|}{ Fabaceae Caesalpinioideae } \\
\hline Copaifera langsdorffii Desf.* & C.lang & copaíba & 110 & 68 & 296 & 474 \\
\hline Sclerolobium paniculatum Vogel & S.pani & tachi & & & 5 & 5 \\
\hline \multicolumn{7}{|l|}{ Fabaceae Faboideae } \\
\hline Acosmium subelegans (Mohlenbr.) Yakovlev & A.sube & peroba-do-campo & 14 & 27 & 1 & 42 \\
\hline Andira anthelmia (Vell.) J.F. Macbr & A.anth & angelim-morcego & & & 3 & 3 \\
\hline Bowdichia virgilioides Kunth & B.virg & sucupira & & & 6 & 6 \\
\hline Dalbergia miscolobium Benth. & D.misc & caviúna-do-cerrado & & & 1 & 1 \\
\hline Machaerium acutifolium Vogel & M.acut & jacarandá-do-campo & 29 & 44 & 31 & 104 \\
\hline Machaerium brasiliense Vogel & M.bras & sapuva & 1 & 1 & 3 & 5 \\
\hline Platypodium elegans Vogel & P.eleg & amendoim-do-campo & 10 & & 32 & 42 \\
\hline \multicolumn{7}{|l|}{ Fabaceae Mimosoideae } \\
\hline Anadenanthera falcata (Benth.) Speg. & A.falc & angico-do-cerrado & 7 & 60 & 9 & 76 \\
\hline Dimorphandra mollis Benth. & D.moll & falso barbatimão & 23 & 19 & & 42 \\
\hline Plathymenia reticulata Benth. & P.reti & vinhático & 2 & 4 & 3 & 9 \\
\hline Stryphnodendron obovatum Benth. & S.obov & barbatimão & 73 & 54 & & 127 \\
\hline \multicolumn{7}{|l|}{ Flacourtiaceae } \\
\hline Casearia lasiophylla Eichler* & C.lasi & cambroé & 1 & & & 1 \\
\hline Lauraceae & & & 186 & 251 & 220 & 657 \\
\hline Nectandra cuspidata Nees \& Mart.* & N.cusp & canela-bosta & & & 71 & 71 \\
\hline Nectandra lanceolata Nees* & N.lanc & canela-amarela & & & 1 & 1 \\
\hline Ocotea corymbosa (Meisn.) Mez.* & O.cory & canelinha & 186 & 250 & 112 & 548 \\
\hline Ocotea velloziana (Meisn.) Mez* & O.vell & canela-branca & & & 3 & 3 \\
\hline Ocotea velutina (Nees) Rohwer* & O.velu & canelão & & & 2 & 2 \\
\hline Persea wildenovii Kosterm* & P.wild & abacateiro-do-mato & & 1 & 31 & 32 \\
\hline \multicolumn{7}{|l|}{ Lythraceae } \\
\hline Lafoensia pacari A.St.-Hil.* & L.paca & dedaleiro & 2 & 1 & & 3 \\
\hline \multicolumn{7}{|l|}{ Malpighiaceae } \\
\hline Byrsonima basiloba A.Juss. & B.basi & murici & & 2 & 1 & 3 \\
\hline Byrsonima intermedia A.Juss. & B.inte & murici-do-campo & & 1 & & 1 \\
\hline Byrsonima laxiflora Griseb. & B.laxi & murici-do-mato & & 13 & 50 & 63 \\
\hline Byrsonima sp. & Byr sp & & & & 6 & 6 \\
\hline \multicolumn{7}{|l|}{ Melastomataceae } \\
\hline Miconia ligustroides (DC.) Naudin* & M.ligu & jacatirão-do-brejo & & 6 & 4 & 10 \\
\hline Miconia sellowiana Cogn.* & M.sell & capa-rosa & & 1 & 1 & 2 \\
\hline \multicolumn{7}{|l|}{ Monimiaceae } \\
\hline Siparuna guianensis Aubl.* & S.guia & limão-bravo & & & 61 & 61 \\
\hline \multicolumn{7}{|l|}{ Moraceae } \\
\hline Ficus guaranitica Chodat* & F.guar & figueira & & & 1 & 1 \\
\hline Pseudolmedia laevigata Trécul* & P.laev & & & & 14 & 14 \\
\hline
\end{tabular}


Tabela 1 - Cont

Table 1 - Cont.

\begin{tabular}{|c|c|c|c|c|c|c|}
\hline \multicolumn{7}{|l|}{ Myrsinaceae } \\
\hline Rapanea ferruginea (Ruiz \& Pav.) Mez & R.ferr & capororoca & & 1 & 15 & 16 \\
\hline Rapanea umbellata (Mart.) Mez.* & R.umbe & capororoca & 5 & 21 & 15 & 41 \\
\hline \multicolumn{7}{|l|}{ Myrtaceae } \\
\hline Eugenia aurata O.Berg & E.aura & & 8 & 4 & 3 & 15 \\
\hline Eugenia livida O.Berg & E.livi & & 1 & 2 & & 3 \\
\hline Eugenia pluriflora DC.* & E.plur & & & 2 & 7 & 9 \\
\hline Gomidesia lindeniana O.Berg* & G.lind & pimenteira & & & 3 & 3 \\
\hline Myrcia bella Cambess.* & M.bell & myrcia & 11 & 21 & 2 & 34 \\
\hline Myrcia aff.lingua (O.Berg) Mattos & M.ling & & 1 & 2 & & 3 \\
\hline Myrcia fallax (Rich.) DC. & M.fall & coração-tinto & & & 3 & 3 \\
\hline Myrcia guianensis (Aubl.) DC.* & M.guia & guamirim-vermelho & 14 & 27 & 12 & 53 \\
\hline Myrcia lingua (O. Berg) Mattos \& D. Legrand & M.ling & brasa-viva & 12 & 2 & & 14 \\
\hline Myrcia multiflora (Lam.) DC.* & M.mult & cambuí & 11 & 11 & 40 & 62 \\
\hline Myrcia prunifolia DC. & M.prun & & 1 & & & 1 \\
\hline Myrcia rostrata DC. & M.rost & guamirim-de-folha-fina & & & 1 & 1 \\
\hline Myrcia venulosa DC.* & M.venu & & 4 & 4 & 14 & 22 \\
\hline Myrciaria delicatula (DC.) O.Berg* & M.deli & araçá-do-mato & 3 & & & 3 \\
\hline Psidium firmum O.Berg & P.pohl & goiabinha-do-cerrado & 3 & & & 3 \\
\hline \multicolumn{7}{|l|}{ Nyctaginaceae } \\
\hline Guapira noxia (Netto) Lundell & G.noxi & maria-faceira & & 1 & 1 & 2 \\
\hline Guapira opposita (Vell.) Reitz* & G.oppo & maria-mole & & & 1 & 1 \\
\hline \multicolumn{7}{|l|}{ Ochnaceae } \\
\hline Ouratea spectabilis (Mart. ex Engl.) Engl. & O.spec & batiputá & 12 & 12 & & 24 \\
\hline \multicolumn{7}{|l|}{ Pinaceae } \\
\hline Pinus elliottii Engelm. & P.elli & pinheiro & & 1 & & 1 \\
\hline \multicolumn{7}{|l|}{ Proteaceae } \\
\hline Roupala montana Aubl.* & R.mont & carne-de-vaca & & & 1 & 1 \\
\hline \multicolumn{7}{|l|}{ Rosaceae } \\
\hline Prunus myrtifolia (L.) Urb.* & P.myrt & pessegueiro-bravo & 2 & & & 2 \\
\hline \multicolumn{7}{|l|}{ Rubiaceae } \\
\hline Amaioua intermedia Mart.* & A.inte & pau-carvão & & & 54 & 54 \\
\hline Faramea montevidensis (Cham. \& Schltdl DC.* & F.mont & pimenteira-do-mato & & & 150 & 150 \\
\hline \multicolumn{7}{|l|}{ Sapindaceae } \\
\hline Matayba elaeagnoides Radlk* & M.elae & camboatã & 1 & & & 1 \\
\hline \multicolumn{7}{|l|}{ Sapotaceae } \\
\hline Pouteria ramiflora (Mart.) Radlk.* & P.rami & abiu-do-cerrado & 2 & 4 & 25 & 31 \\
\hline Pouteria SP & Pou sp & abiurana & & & 1 & 1 \\
\hline \multicolumn{7}{|l|}{ Styracaceae } \\
\hline Styrax camporum Pohl & S.camp & benjoeiro & & & 4 & 4 \\
\hline Styrax ferrugineus Ness \& Mart. & S.ferru & limoeiro-do-mato & & 2 & & 2 \\
\hline Styrax pohlii A. DC. & S.pohl & árvore-de-bálsamo & & & 2 & 2 \\
\hline $\begin{array}{l}\text { Symplocaceae } \\
\text { Symplocos mosenii Brand* }\end{array}$ & S.mose & & & 1 & 4 & 5 \\
\hline $\begin{array}{l}\text { Thymelaeaceae } \\
\text { Daphnopsis fasciculata (Meisn.) Nevling* }\end{array}$ & D.fasc & embira & & 1 & 14 & 15 \\
\hline $\begin{array}{l}\text { Tiliaceae } \\
\text { Tilitis }\end{array}$ & & & & & & \\
\hline Luehea grandiflora Mart.* & L.gran & açoita-cavalo & 1 & & 1 & 2 \\
\hline Vochysiaceae & & & 179 & 315 & 82 & 576 \\
\hline Qualea cordata (Mart.) Spreng. & Q.cord & pau-terrinha & 38 & 20 & 17 & 75 \\
\hline Qualea grandiflora Mart. & Q.gran & pau-terra & 16 & 6 & 12 & 34 \\
\hline Qualea multiflora Mart. & Q.mult & pau-terra & & 1 & 2 & 3 \\
\hline Vochysia tucanorum Mart. & V.tuca & cinzeiro & 125 & 288 & 51 & 464 \\
\hline
\end{tabular}




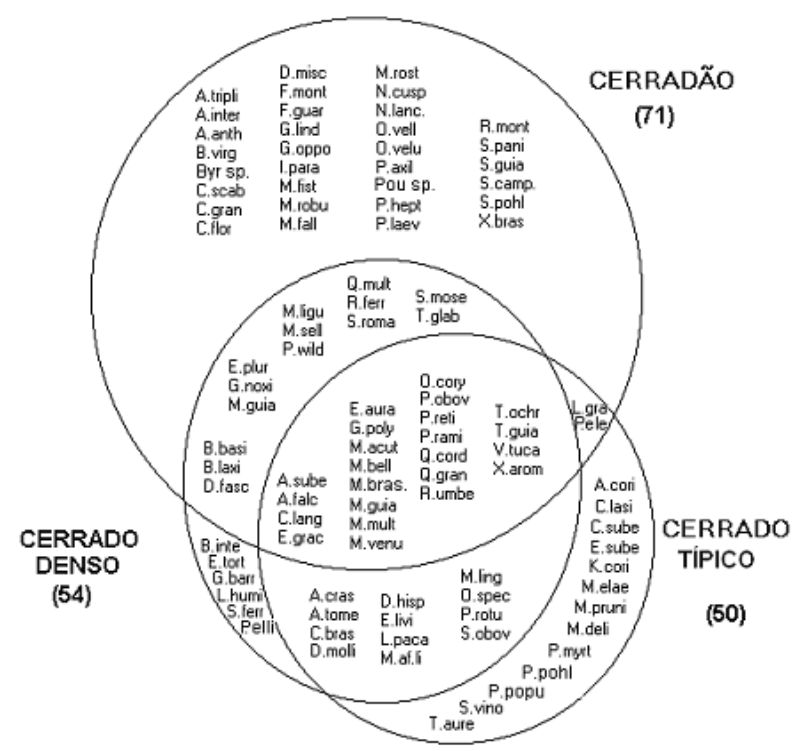

Figura 1 - Distribuição das espécies nas fitofisionomias de Cerrado da Estação Ecológica de Assis, SP (Abreviaturas na Tabela 1).

Figure 1 - Plant species distribution in the Cerrado physiognomies at Assis Ecological Station, state of São Paulo, Brazil (Abbreviations in Table 1).

fitossociológica das comunidades. Quatro espécies, entre as mais importantes no cerradão, são exclusivas dessa fitofisionomia (Protium heptaphyllum, Faramea montevidensis, Nectandra cuspidata e Byrsonima laxiflora), todas elas espécies comuns em remanescentes florestais na região (DURIGAN et al., 2004b).

O dendrograma de similaridade florística (Figura 3) evidencia que o cerradão apresenta composição florística distinta do cerrado típico e do cerrado denso. Há dois grandes grupos de parcelas floristicamente distintos, um reunindo parcelas de cerradão e outro apenas com parcelas de fitofisionomias savânicas (cerrado denso e cerradotípico). Apenas três parcelas (35, 29 e 18) ficaram separadas das demais (similaridade inferior a 0,25), não se enquadrando em nenhum dos grupos. Uma dessas (35) situava-se em uma área ecotonal, em que espécies da floresta estacional se misturavam às do cerrado. As outras duas situavam-se em uma mancha de solos diferenciada do restante da unidade de conservação (DURIGAN, 2011).

Do ponto de vista estrutural, as três fitofisionomias do cerrado na Estação Ecológica de Assis (Tabela 2) mostraram-se distintas. Os resultados da análise de

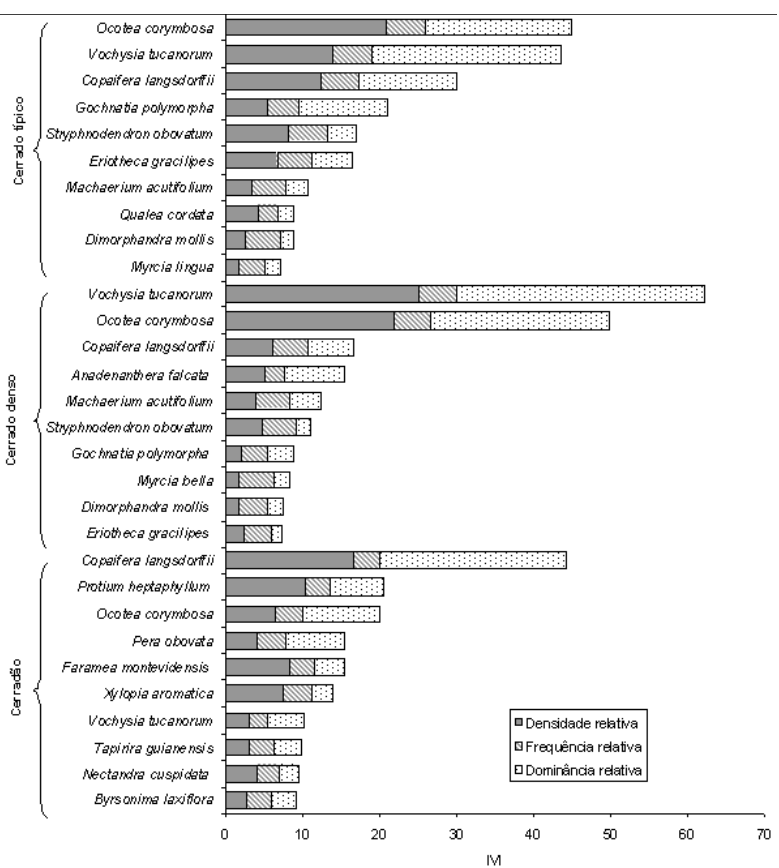

Figura 2 - Parâmetros fitossociológicos das 10 espécies com maior Índice de Valor de Importância (IVI), por fitofisionomia do cerrado na Estação Ecológica de Assis, SP.

Figure 2-Phytosociological parameters of the ten most important species in three cerrado phytophysiognomies at Assis Ecological Station, state of São Paulo, Brazil.

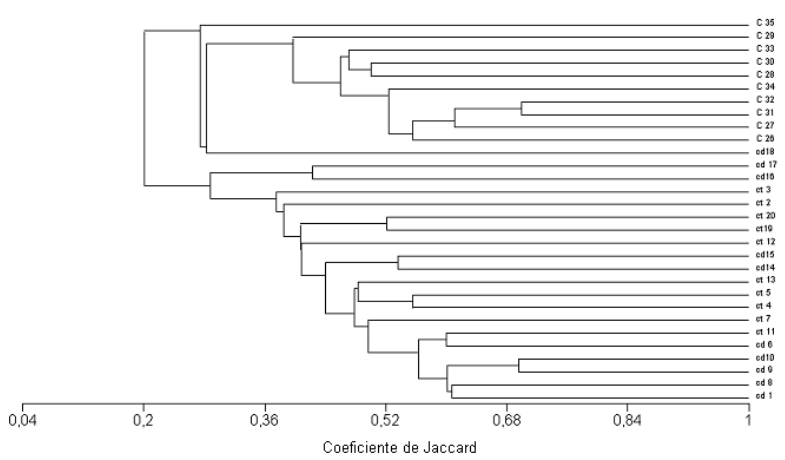

Figura 3 - Dendrograma de análise aglomerativa das espécies que ocorrem no cerradotípico (ct), cerrado denso (cd) e cerradão (C), utilizando-se o coeficiente de similaridade de Jaccard. Números após as letras ct, cd e C representam a parcela amostrada.

Figure 3 - Cluster analysis dendrogram (UPGMA) of typical cerrado, dense cerrado and woodland cerrado species, using Jaccard's similarity index. Numbers after $c t, c d$ and $C$ represent the plot sampled. 
Tabela 2 - Variáveis estruturais da vegetação de cerrados típico e denso e cerradão, na Estação Ecológica de Assis, SP. Table 2 - Structural parameters of the typical cerrado, dense cerrado and woodland cerrado at Assis Ecological Station.

\begin{tabular}{|c|c|c|c|}
\hline Variáveis estruturais & Cerrado típico & Cerrado denso & Cerradão \\
\hline Altura média (m) & $4,8 \pm 1,9 \mathbf{a}$ & $5,7 \pm 2,3 \mathbf{b}$ & $9,7 \pm 2,9 \mathbf{c}$ \\
\hline Área basal $\left(\mathrm{m}^{2} \mathrm{ha}^{-1}\right)$ & $7,8 \pm 2,6 \mathbf{a}$ & $11,5 \pm 2,8 \mathbf{b}$ & $21,4 \pm 2,0 \mathbf{c}$ \\
\hline Densidade (árvores.ha-1) & $897 \pm 36 \mathbf{a}$ & $1.144 \pm 51 \mathbf{a}$ & $1.779 \pm 49 b$ \\
\hline Cobertura de copas (\%) & $47,9 \pm 5,1 \mathbf{a}$ & $69,8 \pm 5,7 \mathbf{b}$ & $86,5 \pm 3,5 c$ \\
\hline Diâmetro equivalente das 5 maiores árvores $(\mathrm{cm})$ & $22,4 \pm 3,2 \mathbf{a}$ & $27,6 \pm 5,5 \mathbf{b}$ & $30,0 \pm 4,7 \mathbf{b}$ \\
\hline Altura das 5 maiores árvores (m) & $6,6 \pm 3,6 \mathbf{a}$ & $11,1 \pm 1,1 \mathbf{b}$ & $16,4 \pm 1,1 \mathrm{c}$ \\
\hline
\end{tabular}

Valores seguidos da mesma letra dentro da linha não diferem entre si, pelo teste de Tukey a 5\% de probabilidade. Values followed by the same letter in the line do not differ by the Tukey's test, considering 5\% of probability.

variância indicaram diferença significativa entre as fitofisionomias, comprovada pelo teste de Tukey (Tabela 2), em quase todas as comparações, excetuando-se a densidade absoluta de árvores no cerrado típico e no cerrado denso e o diâmetro equivalente das cinco maiores árvores no cerrado denso e no cerradão, que não foram significativamente diferentes (Tabela 2). Em todas as outras comparações (área basal, cobertura de copas e altura média de todas as árvores) foi possível distinguir estruturalmente as fitofisionomias do cerrado.

O cerrado típico caracteriza-se por área basal arbórea ao redor de $8 \mathrm{~m}^{2} \mathrm{ha}^{-1}$ e cobertura de copas do estrato arbóreo próxima de 50\%. Aárea basal arbórea no cerrado denso gira em torno de $12 \mathrm{~m}^{2} \mathrm{ha}^{-1}$,e as copas das árvores cobrem cerca de $70 \%$ do terreno. No cerradão, a área basal arbórea fica próxima de $20 \mathrm{~m}^{2} \mathrm{ha}^{-1}$ e a cobertura de copas, ao redor de $90 \%$.

\section{DISCUSSÃO}

Embora o valor da diversidade florística do cerradão tenha sido semelhante, ou até superior (TOPPA et al., 2006; PINHEIRO, 2006), aos obtidos em outras localidades, ele não ocorreu nas fitofisionomias savânicas. Para estas, a diversidade florística obtida neste estudo foi relativamente baixa, se comparada com os valores mencionados sobre outras áreas de cerrado no Estado de São Paulo por Martins e Santos (1999) - 2,98, Durigan et al. (2002) - 3,02, Fidelis e Godoy (2003) - 3,62, Teixeira et al. (2004) - 3,05, Pereira-Silva et al. (2004) - 3,47 e Gomes et al. (2004) - 3,38.

A explicação para a baixa diversidade das fitofisionomias savânicas na área de estudo pode estar na localização marginal em relação à área de domínio do cerrado, no seu limite austral, onde as taxas de extinção local de espécies podem ter sido maiores, mediante o gradual desaparecimento regional das fisionomias savânicas (ROSSATO et al., 2008; PINHEIRO; DURIGAN, 2009). O adensamento da vegetação de cerrado em diversas regiões do Estado de São Paulo (DURIGAN et al., 1987; RATTER et al., 1988; PINHEIRO, 2006; DURIGAN; RATTER, 2006; PINHEIRO; DURIGAN, 2009), inclusive na região deste estudo, indica que, ao cessarem as pressões antrópicas, as fitofisionomias savânicas tendem a desaparecer, com o ambiente tornando-se mais favorável às espécies florestais. E isso pode ser uma razão a mais para explicar a baixa diversidade do cerrado típico e do cerrado denso, também observada por Pinheiro (2006) em Corumbataí, SP. Diferenças de riqueza e diversidade florística entre locais, no entanto, devem ser vistas com ressalvas, pois podem ser explicadas, entre outros fatores, por diferenças no critério de inclusão e universo amostral. O índice de diversidade de Shannon é sensível ao critério de inclusão (MARTINS; SANTOS, 1999), de modo que a comparação entre estudos incorpora os efeitos de diferenças no número de indivíduos e espécies amostradas em cada caso.

Do total de espécies amostradas, 23\% estiveram presentes com apenas um indivíduo e, naturalmente, aparecem como exclusivas de uma ou outra fitofisionomia, mas podem não ter sido amostradas em outras fisionomias apenas por serem muito raras. Por isso, foram discutidas as espécies exclusivas com base apenas nas mais abundantes.

Na tentativa de explicar as diferenças florísticas entre as fitofisionomias, de acordo com a lista de espécies e suas características ecológicas, constata-se, com base na classificação das espécies da flora regional (DURIGAN et al., 2004b), que todas as espécies exclusivas do cerradão que tiveram pelo menos 10 árvores amostradas são tolerantes à sombra (Amaioua intermedia, Faramea montevidensis, Nectandra

Revista Árvore, Viçosa-MG, v.36, n.1, p.181-193, 2012 
cuspidata, Pseudolmedia laevigata, Protium heptaphyllum e Siparuna guianensis). No entanto, as espécies que ocorrem apenas nas fitofisionomias savânicas (cerrado denso e cerrado típico), com mais de 10 árvores, não se desenvolvem à sombra.

Para Franco (2005), os diferentes níveis de sombreamento podem definir a sucessão de espécies na paisagem. Aquelas tolerantes à sombra ocorrem em formações florestais (cerradão), enquanto espécies heliófilas, possuidoras de mecanismos capazes de resistir aos efeitos da incidência sem restrição de radiação solar, predominam em ambientes mais abertos. O processo de adensamento da vegetação observado por Durigan e Ratter (2006) e Pinheiro e Durigan (2009) na região deste estudo permite supor que, nas áreas ocupadas por cerradão, espécies heliófilas exclusivas das fitofisionomias savânicas teriam sido eliminadas da comunidade e estariam sendo substituídas por espécies tolerantes à sombra, mas que não se desenvolvem satisfatoriamente no ambiente seco e ensolarado das fitofisionomias savânicas.

A surpreendente riqueza de espécies registrada nas parcelas de cerradão, maior do que nas fisionomias savânicas e contrariando o que vem sendo observado em outros locais (COSTA et al., 2010), tem na proximidade de fragmentos florestais a explicação mais provável no caso da área de estudo. Muitas espécies amostradas ocorrem tanto no cerrado quanto na Floresta Estacional Semidecidual na região (DURIGAN et al., 2004b), e a fauna dispersora de sementes pode estar adicionando espécies florestais ao cerradão. Essa hipótese encontra respaldo nas análises de similaridade florística entre as fitofisionomias, que mostram que o cerradão possui composição florística distinta do cerrado típico e do cerrado denso. Esse resultado corrobora a observação de Ribeiro e Walter (1998) de que, em termos florísticos, as formações savânicas apresentam maior semelhança entre si do que em relação ao cerradão.

A estrutura florestal, somada à diferenciação florística, coloca em dúvida a classificação dos cerradões paulistas como uma das fisionomias do gradiente que forma o cerrado lato sensu. As transformações pelas quais essa vegetação vem passando nas últimas décadas, após a supressão do fogo e do pastoreio (DURIGAN; RATTER, 2006; PINHEIRO; DURIGAN, 2009), parecem configurar uma etapa sucessional do processo de expansão das florestas sobre as savanas, que vem ocorrendo desde o início do Holoceno no Sudeste do Brasil (BEHLING, 1998).

Apesar de haver diferenças estruturais significativas entre as fitofisionomias em estudo, essas diferenças são menos acentuadas entre o cerrado típico e o cerrado denso, quando comparados com o cerradão. É senso comum que as fitofisionomias do cerrado não são claramente separáveis em categorias, formando, na realidade, um gradiente estrutural (OLIVEIRA FILHO; RATTER, 2002; RIBEIRO; WALTER, 2008), e estudos recentes apontam que a estrutura da vegetação do cerrado se modifica no tempo (DURIGAN; RATTER, 2006; PINHEIRO; DURIGAN, 2009). Todavia, a classificação das fitofisionomias do cerrado é desejável para dar suporte a estudos ecológicos e, também, para a cartografia de tipos vegetacionais em escalas mais detalhadas. Os resultados deste estudo indicam que é possível classificar as fitofisionomias do cerrado em dado momento no tempo, considerando-se, como centro de classes, os valores médios dos descritores estruturais quantitativos obtidos de cada fitofisionomia.

Uma vez que a densidade absoluta e a altura média de todas as árvores são dependentes do critério de inclusão e que o porte das maiores árvores não foi claramente distinto entre os tipos de vegetação estudados, a área basal, expressa $\mathrm{em} \mathrm{m}^{2}$.ha ${ }^{-1}$, e a cobertura de copas do estrato arbóreo (\%) colocam-se como os melhores descritores para a classificação das fitofisionomias do cerrado. A área basal de comunidades arbóreas, por ser muito pouco influenciada pelo critério de inclusão (DURIGAN, 2009), uma vez que na sua quase totalidade é composta pelas árvores adultas, e por ser facilmente mensurável, além de globalmente utilizada, possibilitando comparações, pode ser considerada a melhor variável para classificar fitofisionomias do cerrado. Os valores médios de altura e diâmetro das maiores árvores em cada fitofisionomia podem ser utilizados como ferramentas complementares de classificação.

\section{CONCLUSÕES}

Há uma clara diferenciação florística do cerradão em relação às fisionomias savânicas (cerrado típico e cerrado denso) que não diferem entre si pelas espécies que as compõem.

Revista Árvore, Viçosa-MG, v.36, n.1, p.181-193, 2012

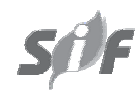


As três fitofisionomias de cerrado estudadas mostraram-se estruturalmente distintas em classes de área basal, cobertura de copas, diâmetro e altura das maiores árvores.

O melhor descritor para classificar as fitofisionomias, por ser facilmente mensurável e pouco variável com o critério de inclusão, é a área basal $\left(\mathrm{m}^{2} \cdot \mathrm{ha}^{-1}\right)$. A cobertura de copas e os valores médios de altura e diâmetro das maiores árvores complementam os critérios para a classificação fitofisionômica da vegetação do Cerrado.

\section{AGRADECIMENTOS}

Aos funcionários da Floresta Estadual de Assis Edivaldo Furlan e Nelson Keki e aos ajudantes de campo Fernando Souza e Douglas Zimmermann, pelo suporte durante os levantamentos; ao Conselho Nacional do Desenvolvimento Científico e Tecnológico (CNPq), pelo auxílio à pesquisa (Processo 475286/2007-3 ) e pela concessão das bolsas de doutorado a E. S. Pinheiro e de produtividade em pesquisa a G. Durigan.

\section{REFERÊNCIAS}

AGUIAR, L. M. S.; MACHADO, R. B.; MARINHO-FILHO, J. A diversidade biológica do Cerrado. In: AGUIAR, L. M. S.; CAMARGO, A. J. A. (Eds.) Cerrado: ecologia e caracterização. Brasília: Embrapa, 2004. p.17-40

ANGIOSPERM PHYLOGENY GROUP - APG. An update of the angiosperm phylogeny group classication for the orders and families of flowering plants: APG II. Botanical Journal of the Linnean Society, v.141, p.399-436, 2003.

BALDUINO, A. P. C. et al. Fitossociologia e análise comparativa da composição florística do cerrado da flora de Paraopeba-MG. Revista Árvore, v.29, n.1, p.25-34, 2005.

BEHLING, H. Late quaternary vegetational and climatic changes in Brazil. Review of Palaeobotany and Palinology, v.99, n.2, p.143-156, 1998

BELBIN, L.; MCDONALD, C. Comparing three classification strategies for use in ecology. Journal of Vegetation Science, v.4, n.3, p.341-348, 1993.
BRANDO, P. M.; DURIGAN, G. Changes in cerrado vegetation after disturbance by frost (São Paulo State, Brazil). Plant Ecology, v.175, n.2, p.205-215, 2004.

CANFIELD, R. H. Application of the line interception method in sampling range vegetation. Journal of Forestry, v.39, n.4, p.388-394, 1941.

COSTA, C. P., CUNHA, C. N.; COSTA, S. C. Characterization of the flora and structure of the shrub-tree layer in a cerrado area in Pantanal do Poconé, MT. Biota Neotropica, v.10, n.3, p.61-73, 2010.

COUTINHO, L. M. O conceito de cerrado. Revista Brasileira de Botânica, v.1, n.1, p.17-24, 1978.

DURIGAN, G. Métodos para análise de vegetação arbórea.. In: CULLEN Jr., L.; RUDRAN, R.; PADUA-VALLADARES, C. (Orgs.) Métodos de estudos em biologia da conservação e manejo da vida silvestre. Curitiba: UFPR/ Fundação o Boticário de Proteção à Natureza, 2003. p.455-479.

DURIGAN, G. Observations on the southern cerrados and their relationship with the core are. In: PENNINGTON, R. T.; LEWIS, G. P.; RATTER, J. A. (Eds.) Neotropical savannas and seasonally dry forests: plant diversity, biogeography and conservation. London: Taylor e Francis, 2006. p.67-77.

DURIGAN, G. Estrutura e diversidade de comunidades florestais.. In: MARTINS, S. V. (Ed.) Ecologia de florestas tropicais do Brasil. Viçosa, MG: Universidade Federal de Viçosa, 2009. p185-215.

DURIGAN, G. Plano de manejo da Estação Ecológica de Assis. São Paulo: Instituto Florestal, 2010. 172p. Disponível em: $<$ http://www.iflorestal.sp.gov.br/ Plano_de_manejo/index.asp $>$. Acesso em: 21 de abr. de 2011.

DURIGAN, G. et al. Inventário florístico do cerrado na Estação Ecológica de Assis, SP. Hoehnea, v.26, p.149-172, 1999.

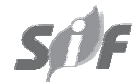

Revista Árvore, Viçosa-MG, v.36, n.1, p.181-193, 2012 
DURIGAN, G.; FRANCO, G. A. D. C.; SIQUEIRA, M. F. A vegetação dos remanescentes de cerrado no estado de São Paulo. In: BITENCOURT, M. D.; MENDONÇA, R. R. (Orgs.) Viabilidade de conservação dos remanescentes de cerrado no estado de São Paulo. São Paulo: Annablume, FAPESP, 2004a. p.29-56.

DURIGAN, G. et al. Caracterização de dois estrados da vegetação em uma área de cerrado no município de Brotas, SP, Brasil. Acta

Botanica Brasilica, v.16, n.3, p.251-262, 2002.

DURIGAN, G.; RATTER, J. A. Successional changes in cerradão and cerrado/forest ecotonal vegetation in western São Paulo State, Brazil, 1962-2000. Edinburgh Journal of Botany, v.63, n.1, p.119-130, 2006.

DURIGAN, G. et al. Fitossociologia e evolução da densidade da vegetação do cerrado de Assis, SP.

Boletim Técnico do Instituto Florestal, v.41, n.1, p.59-78, 1987.

DURIGAN, G. et al. Flora arbustiva-arbórea do médio Paranapanema: base para a restauração dos ecossistemas naturais. In: VILAS BOAS, O.; DURigan, G. (Orgs.) Pesquisas em conservação e recuperação ambiental no oeste paulista: resultados da cooperação Brasil/Japão. São Paulo: Secretaria do Meio Ambiente/IF/JICA, 2004b. p.199-239.

EITEN, G. Formas fisionômicas do Cerrado. Revista Brasileira de Botânica, v.2, n.2, p.139-148, 1979.

EITEN, G. The cerrado vegetation of Brazil. Botanical Review, v.38, n.2, p.201-341, 1972.

FELFILI, J. M. et al. (Eds.) Projeto biogeografia do bioma cerrado: vegetação e solos.

Caderno de Geociências do IBGE, v.12, p.75-166, 1994.

FIDELIS, A. T.; GODOY, S. A. P. Estrutura de um cerrado stricto sensu na gleba cerrado Péde-Gigante, Santa Rita do Passa Quatro, SP. Acta Botanica Brasilica, v.17, n.4. p.531-539, 2003.
FRANCO, A. Biodiversidade de forma e função: implicações ecofisiológicas das estratégias de utilização de água e luz em plantas lenhosas do Cerrado.. In: SCARIOT, A.; SOUZA-SILVA, J. C.; FELFILI, J. M. (Orgs.) Cerrado: ecologia, biodiversidade e conservação. Brasília: Ministério do Meio Ambiente, 2005. p.179-196

GOMES, B. Z.; MARTINS, F. R.; TAMASHIRO, J. Y. Estrutura do cerradão e da transição entre cerradão e floresta paludícola num fragmento da International Paper do Brasil Ltda., Brotas, SP. Revista Brasileira de Botânica, v.27, n.2, p.249-262, 2004.

JAMES, F. C.; MCCULLOCH, C. E. Multivariate analysis in ecology and systematics: Panacea or Pandora's box. Annual. Ecology and Systematics, v.21, p.129-166,1990.

MACHADO, R. B. et al. Estimativas de perda de área de Cerrado brasileiro. Relatório técnico não publicado. Brasília: Conservação Internacional, 2004.

MARTINS, F. R.; SANTOS, F. A. M. Técnicas usuais de estimativa da biodiversidade. Revista Holos, v.1, p.236-267, 1999.

MELO, A. C. G.; MIRANDA, D. L. C.; DURIGAN, G. Cobertura de copas como indicador de desenvolvimento estrutural de reflorestamentos de restauração de matas ciliares no Médio Vale do Paranapanema, SP, Brasil. Revista Árvore, v.31, n.2, p.321-328, 2007.

MÜELLER-DOMBOIS, D.; ELLENBERG, H. Aims and methods in vegetation ecology. New York: John Wiley \& Sons, 1974. 547p.

MYERS, N. et al. Biodiversity hotspots for conservation priorities. Nature, v.403, p.853858, 2000.

OLIVEIRA-FILHO, A. T.; RATTER, J. A. Vegetation physiognomies and woody flora of the Cerrado biome. In: OLIVEIRA, P. S.; MARQUIS, R. J. (Eds.) The cerrados of Brazil: ecology and natural history of a neotropical savanna. New York: Columbia University, Columbia University Press, 2002. p. 91-120. 
PEREIRA-SILVA, E. F. L. et al. Florística e fitossociologia dos estratos arbustivo e arbóreo de um remanescente de cerradão em uma unidade de conservação do estado de São Paulo.

Revista Brasileira de Botânica, v.27, p.533-544, 2004.

PIELOU, E. C. Ecological diversity. New York: John Wiley \& Sons, 1975. 165p.

PIELOU, E. C. Species diversity and pattern diversity in the study of ecological succession. Journal of Theo-retical Biology , v.10, n.2, p.370-383, 1966.

PINHEIRO, E. S.; DURIGAN, G. Dinâmica espaçotemporal (1962-2006) das fitofisionomias em unidade de conservação do Cerrado no sudeste do Brasil. Revista Brasileira de Botânica, v.32, n.3, p.441-454, 2009.

PINHEIRO, M. H. O. Composição e estrutura de uma comunidade savânica em gradiente topográfico no município de Corumbataí (SP, Brasil). 2006. 119f. Tese (Doutorado em Ciências Biológicas) - Universidade Estadual Paulista “Julio de Mesquita Filho”, Rio Claro, 2006.

RATTER, J. A.; BRIDGEWATER, S.; RIBEIRO, F. Biodiversity patterns of the woody vegetation of the Brazilian Cerrado. In: PENNINGTON; R.T.; LEWIS, G.P.; RATTER, J.A. (Eds.) Neotropical savannas and seasonally dry forests: plant diversity, biogeography and conservation. London, Taylor e Francis, 2006. p.31-66.

RATTER, J. A. et al. Floristic composition and community structure of a Southern cerradoarea in Brazil. Notes of the Royal Botanical Garden of Edinburg, v.45, n.1, p.137-151, 1988.

RIBEIRO, J. F.; WALTER, B. M. T. As principais fitofitofisionomias do bioma Cerrado. In: SANO, S. M.; ALMEIDA, S. P.; RIBEIRO, J. F. (Eds.). Cerrado: ecologia e flora. Brasília: Embrapa, 2008. p.151-212.
RIBEIRO, J. F. et al. Os principais tipos fitofisionômicos da região dos Cerrados. Brasília: Embrapa, 1983. 28p. (Boletim de Pesquisa, 21)

RIBEIRO, J. F.; SILVA, J. C. S.; BATMANIAN, G. J. Fitossociologia de tipos fisionômicos de cerrado em Planaltina, DF. Revista Brasileira de Botânica, v.8, n.2, p.131-142, 1985.

RIBEIRO, J. F.; WALTER, B. M. T.

Fitofitofisionomia do Bioma Cerrado. In: SANO, S. M.; ALMEIDA, S. P. (Eds.) Cerrado: ambiente e flora. Brasília: Embrapa, 1998. p.89-166.

ROSSATTO, D. R.; TONIATO, M. T. Z.; DURIGAN, G. Flora fanerogâmica não-arbórea do cerrado na Estação Ecológica de Assis, Estado de São Paulo Revista Brasileira de Botânica, v.31, n.3, p.409-424, 2008.

TEIXEIRA, M. I. J. G. et al. Florística e fitossociologia de área de cerrado s.s no município de Patrocínio Paulista, nordeste do estado de São Paulo. Bragantia, v.63, n.2, p.1-11, 2004.

TOPPA, R. H. et al. Mapeamento e caracterização das fitofitofisionomias da Estação Ecológica de Jataí. In: SANTOS, J. E.; PIRES, J. S. R.; MOSCHINI. L. E. (Orgs.) Estudos integrados em ecossistemas: Estação Ecológica de Jataí. São Carlos: Rima, 2006. p.21-44.

VENN, J. On the diagrammatic and mechanical representation of propositions and reasonings". Dublin Philosophical Magazine and Journal of Science, v.10, n.59, p.1-18, 1980.

WALTER, B. M. T. Fitofitofisionomias do Bioma Cerrado: síntese terminológica e relações florísticas. 2006. 389f. Tese (Doutorado em Ecologia) - Universidade de Brasília, Brasília, 2006.

ZAR, J. H. Biostatistical analysis. 4.ed. New Jersey: Prentice Hall, 1999. 663p. 